\title{
Efecto de la temperatura en el módulo dinámico del cemento asfáltico y la mezcla asfáltica - caso de Boyacá
}

\section{Effect of temperature on dynamic modulus of asphalt cement and asphalt mixture - case Boyacá}

\section{Efeito da temperatura no módulo dinâmico do cimento asfáltico e da mistura asfáltica - caso de Boyacá}

\author{
Carlos Hernando Higuera-Sandoval ${ }^{1}$
}

Forma de citar: C. H. Higuera- Sandoval, "Efecto de la temperatura en el módulo dinámico del cemento asfáltico y la mezcla asfáltica - caso de Boyacá”, Respuestas, vol. 21, no. 1, pp. 108-119, 2016.

Recibido:

Marzo 18 de 2015

Aceptado:

Julio 30 de 2015
${ }^{1}$ Magister en Ingeniería Docente Titular - Escuela de Transporte y Vías Universidad Pedagógica y Tecnológica de Colombia carlos.higuera@uptc.edu.co Orcid: 0000-0003-1333-2517 Tunja-Colombia

\section{Resumen}

Objetivo: El presente artículo muestra de manera detallada el efecto de la temperatura en la determinación del módulo dinámico del cemento asfáltico y el módulo dinámico de la mezcla asfáltica. Método: Para la investigación se consideraron las temperaturas medias anuales ponderadas más representativas de las regiones del departamento de Boyacá de $13^{\circ} \mathrm{C}$ a $20^{\circ} \mathrm{C}$, también la velocidad de operación de sus carreteras de 30, 50, 70 y 100 $\mathrm{Km} /$ hora y se analizaron dos cementos asfálticos del tipo AC 60 - 70 y AC 80 - 100, estipulados en el artículo INV 400-13 de las especificaciones generales de construcción de carreteras del Instituto Nacional de Vías - INVIAS - 2013 y que son de uso frecuente en la construcción de pavimentos en el departamento de Boyacá. Para la determinación de los módulos dinámicos del asfalto y de la mezcla asfáltica se siguió la metodología de la SHELL y se analizaron las variables como la temperatura media anual ponderada del aire - TMAP, la temperatura de trabajo de la mezclas - tmix, la velocidad de operación de las carreteras - Vop, el tiempo de aplicación de la carga - t, la frecuencia de la aplicación de la carga - F y el índice de penetración del asfalto - IP. Resultados: Se presentan los módulos dinámicos del asfalto y de la mezcla asfáltica para diferentes temperaturas medias anuales ponderadas y diferentes velocidades de operación de las carreteras del Departamento de Boyacá, para los asfaltos tipo AC 60-70 y AC 80-100. Conclusión: El aporte de este trabajo de investigación es de gran utilidad para los ingenieros de diseño de estructuras de pavimentos, porque permite conocer el módulo dinámico de una mezcla asfáltica densa en caliente tipo INVIAS MDC-19 para rodadura, teniendo en cuenta la temperatura media anual ponderada de la zona del proyecto y las características de operación de la carretera, variables fundamentales para el diseño de las estructuras de pavimento flexible.

Palabras clave: Diseño de pavimentos flexibles, mecánica de pavimentos, mezclas asfálticas, módulos dinámicos.
Abstract
Objective: This article shows in detail the effect of temperature on the determination of the dynamic module of asphaltic cement and the dynamic module of the asphalt mix. Method: Average pondered annual temperatures of the most representative Regions of the department of Boyaca ranging $13^{\circ} \mathrm{C}$ to $20^{\circ} \mathrm{C}$ were considered, as well as the speed of operation of its highways $30,50,70$ and $100 \mathrm{~km} /$ hour and were considered, and two asphalt cements the type AC 60 - 70 and AC 80 - 100 which are stipulated in the article INV 400-13 of the general specifications for road construction of the National roads Institute - INVIAS - 2013 of frequent use in the pavement construction in the department 
of Boyaca. For the determination of the dynamic modules of asphalt and asphalt mixture the methodology SHELL was followed and variables as the weighted average annual air temperature TMAP, temperature Working Mixtures - TMIX, Speed Operation of roads - VOP, the application time load - $t$, the frequency of application of the load - F and the rate of penetration asphalt - IP were analyzed. Results: Dynamic modules asphalt and asphalt mixture paragraph for different annual pondered average temperatures and different speeds of operation of the roads on the Department of Boyaca are presented, for asphalts type AC 60-70 and AC 80-100. Conclusion: The contribution of this research work is very useful for Design Engineers of pavement structures as it allows to know the dynamic module of a hot-dense asphalt mixture of the type INVIAS MDC-19 for rolling, taking into account the annual pondered average temperature of the project area and the operating characteristics of the road, fundamental variables for the design of flexible pavement structures.

Keywords: Flexible Pavement Design, Mechanics of Pavement, Asphalt Mixtures, dynamic modules.

\section{Resumo}

Objetivo: O presente artigo mostra de maneira detalhada o efeito da temperatura na determinação do módulo dinâmico do cimento asfáltico e o módulo dinâmico da mistura asfáltica. Método: Nesta pesquisa foram consideradas as temperaturas médias anuais ponderadas mais representativas das regiões do Estado de Boyacá, que variam entre $13^{\circ} \mathrm{C}$ e $20^{\circ} \mathrm{C}$, também a velocidade de operação de suas estradas de $30,50,70$ y 100 $\mathrm{km} /$ hora e se analisaram dois cimentos asfálticos do tipo AC $60-70$ e AC $80-100$, estipulados no artigo INV 400-13 das especificações gerais de construção de estradas do Instituto Nacional de Vias - INVIAS - 2013 e que são de uso frequente na construção de pavimentos no Estado de Boyacá. Para a determinação dos módulos dinâmicos do asfalto e da mistura asfáltica se seguiu a metodologia da SHELL e se analisaram as variáveis como a temperatura média anual ponderada do ar - TMAP, a temperatura de trabalho da mistura - tmix, a velocidade de operação das estradas - Vop, o tempo de aplicação da carga - t, a frequência da aplicação da carga - F e o índice de penetração do asfalto - IP. Resultados: Apresentam-se os módulos dinâmicos do asfalto e da mistura asfáltica para diferentes temperaturas médias anuais ponderadas e diferentes velocidades de operação das estradas do Estado de Boyacá, para os asfaltos tipo AC 60-70 e AC 80-100. Conclusão: $\mathrm{O}$ aporte deste trabalho de pesquisa é de grande utilidade para os engenheiros de design de estruturas de pavimentos, devido a que permite conhecer o módulo dinâmico de uma mistura asfáltica densa em quente tipo INVIAS MDC-19, para laminação, tendo em conta a temperatura média anual ponderada da área do projeto e as características de operação da estrada, variáveis fundamentais para o design das estruturas de pavimento flexível.

Palavras-chave: Design de pavimentos flexíveis, mecânica de pavimentos, mistura asfáltica, módulos dinâmicos.
Enero - Junio 2016 ISSN 0122-820X

E-ISSN 2422-5053

PP: 108-119

\section{Introducción}

El Departamento de Boyacá cuenta con varias regiones con climas diferentes y la infraestructura de carreteras con diferente jerarquía vial y velocidades de operación. Las estructuras de pavimentos predominantes se construyen en pavimentos flexibles o asfálticos. Por esta razón, es necesario conocer el comportamiento de las capas asfálticas para las diferentes regiones del departamento de Boyacá y tener parámetro que ayuden al prediseño de las estructuras de pavimento.

El objetivo del presente trabajo de investigación es conocer el efecto de la temperatura en el módulo dinámico del cemento asfáltico y de la mezcla asfáltica en 
No. 1

Enero - Junio 2016 ISSN 0122-820X E-ISSN 2422-5053 PP: 108-119 la modelación de las estructuras de pavimento flexible y dar recomendaciones para su prediseño, evaluación y comportamiento. Para la modelación del efecto de la temperatura en el módulo dinámico del cemento asfáltico y de la mezcla asfáltica se analizaron las metodologías de la SHELL [1]-[4] AASHTO, Heukelom y Klomp, Bonnaure, Instituto del Asfalto y Witczak y se decidió trabajar con las metodologías SHELL y AASHTO debido a su gran correlación y simplicidad para la modelación y el conocimiento en la región de Boyacá.

Para la modelación se tomaron las temperaturas medias mensuales representativas del departamento de Boyacá de $13^{\circ} \mathrm{C}$ a $20^{\circ} \mathrm{C}$, las velocidades de operación de 30, 50, 70 y $100 \mathrm{Km} /$ hora y dos tipos de asfaltos el AC $60-70$ y el AC $80-100$, los cuales son muy utilizados en la fabricación de mezclas asfálticas en el departamento de Boyacá. La modelación permite calcular el tiempo y la frecuencia de aplicación de la carga, el índice de penetración, el módulo dinámico del asfalto y el módulo dinámico de la mezcla asfáltica.

\section{Materiales y métodos}

2.1 Generalidades. Los métodos modernos de diseño de pavimentos exigen como datos de entrada las características elásticas y dinámicas de las capas asfálticas: el módulo dinámico, la relación de Poisson y la ley de fatiga o de comportamiento, las cuales se pueden obtener sobre probetas elaboradas en laboratorio o extraídas directamente de cada una de las capas de la estructura del pavimento.

El módulo dinámico es la relación entre el esfuerzo aplicado bajo carga repetida (condición dinámica) y la deformación elástica recuperable y se determina en (1):

$E_{d}=\frac{\sigma_{d}}{\epsilon_{r}}$

Donde:

$E_{d}: \quad$ Módulo dinámico de la mezcla asfáltica.

$\sigma_{d}: \quad$ Esfuerzo desviador, el cual es el esfuerzo axial en el ensayo de compresión inconfinada.

$\varepsilon_{r}$ : $\quad$ Deformación elástica recuperable.

La Figura 1, muestra el comportamiento de la deformación medida en una probeta sometida a carga repetida. En las aplicaciones iníciales de carga toda la deformación es de tipo plástico o permanente y acumulable, a medida que se aumentan los ciclos de carga, la deformación plástica es menor, al final por ejemplo, después de 100 o 200 ciclos de carga como ocurre en los suelos, solamente se presentará deformación elástica $\left(\mathrm{e}_{\mathrm{r}}\right)$.

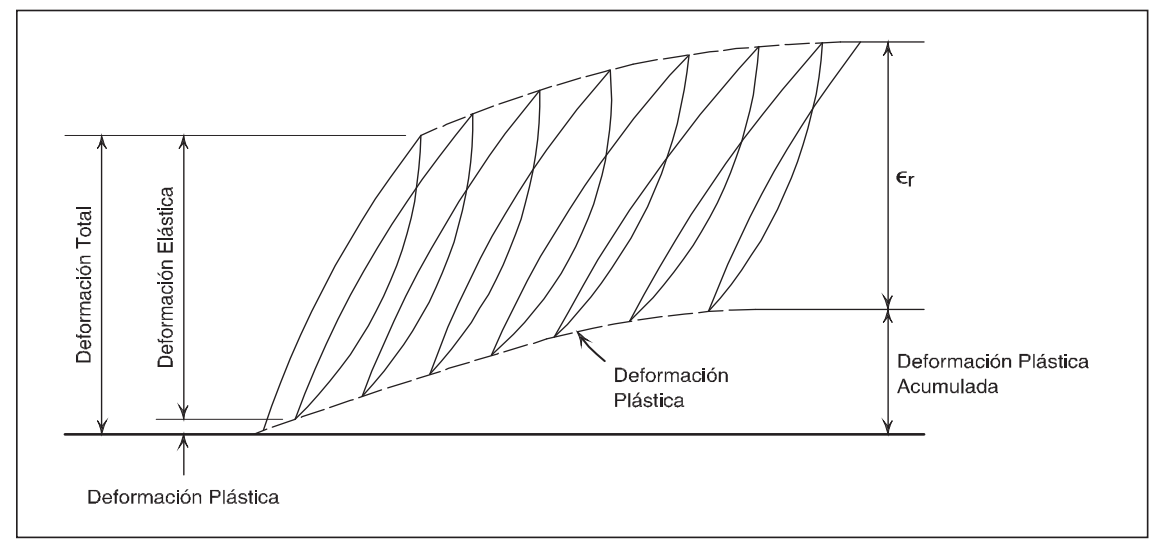

Figura 1. Comportamiento de la deformación medida en una probeta sometida a carga repetida Fuente: Y.H. HUANG, "Material Characterizarion" in Pavement analysis and design, 2th edition. Pearson Prentice Hall, 2004, ch. 7, pp. 280. Fuente: [3]. 
En mezclas asfálticas, teniendo en cuenta la susceptibilidad térmica y las cargas de los vehículos, el valor del módulo dinámico dependerá de la temperatura de la mezcla (T) y del tiempo de aplicación de la carga (t) y se determina mediante (2).

$E_{d}(T, t)=\frac{\sigma_{d}}{\epsilon_{r}}$

Se conocen las siguientes formas de determinar los módulos dinámicos de las mezclas asfálticas [3]:

- Mediante ensayos de laboratorio.

- Mediante fórmulas y correlaciones

- Mediante nomogramas

- Retrocálculo o cálculo inverso

Para el estudio de modelación del comportamiento del módulo dinámico del asfalto y de la mezcla asfáltica se utilizó el método de las correlaciones y se detalla a continuación [1] para el diseño de pavimentos flexibles.

\subsection{Determinación de la temperatura media} anual ponderada del aire - TMAP. Para determinar la TMAP se parte de la información de las temperaturas medias mensuales del aire - TMM de la zona más cercana al proyecto. Generalmente, los análisis se realizan con datos de más de 10 años para poder evaluar efectivamente el comportamiento de la temperatura en la zona del proyecto, la cual influye directamente en el comportamiento estructural y funcional de los pavimentos. Esta información es suministrada por el Instituto de Hidrología, Meteorología y Estudios Ambientales de Colombia - IDEAM. [2].

El procedimiento consiste en el desarrollo de los siguientes pasos:

- Seleccionar las estaciones meteorológicas más cercanas al proyecto y consultar en el IDEAM las temperaturas medias mensuales del aire - TMM de la serie histórica.
- Para cada temperatura media mensual del aire - TMM determinar su factor de ponderación - $f p$, el cual puede calcularse por medio de (3) y (4) o con ayuda del programas de cálculo.

$$
\begin{aligned}
& T M M^{\circ} C=20.348+17.5683 \log (f p) \\
& f p=10^{\left[\frac{T M M^{\circ} C-20.348}{17.5683}\right]}
\end{aligned}
$$

Enero - Junio 2016 ISSN 0122-820X E-ISSN 2422-5053 PP: 108-119

- Se determina el factor de ponderación promedio del año - fprom y con ayuda de (5).

$$
\text { fprom }=\frac{\Sigma f p}{n}
$$

- Se determina la TMAP para cada año con ayuda de (6).

$$
T M A P^{\circ} C=20.348+17.5683 \log (\text { fprom })
$$

- Con la TMAP de los años de la serie histórica se determina la TMAP de diseño como el promedio de las TMAP anuales de la serie histórica.

Donde:

$$
\begin{aligned}
& \text { TMM = Temperatura media mensual del } \\
& f p \quad=\text { Factor de ponderación } \\
& \text { fprom = Factor de ponderación promedio } \\
& T M A P=\text { Temperatura media anual } \\
& \text { ponderada del aire, }{ }^{\circ} \mathrm{C} \\
& n \quad \text { = Número de meses del año }
\end{aligned}
$$

Para este trabajo de investigación se consideró la temperatura media anual ponderada del aire de $13^{\circ} \mathrm{Ca} 20^{\circ} \mathrm{C}$, por ser las más representativas de las regiones del Departamento de Boyacá.

2.3 Determinación de la temperatura de la mezcla- Tmix. La temperatura de la mezcla asfáltica se determina en función de la 
temperatura media anual ponderada de diseño - TMAP y se realizó con ayuda del ábaco RT de la SHELL.

Para el presente trabajo de investigación se calculó la Tmix en función de la TMAP más representativas de las regiones del Departamento de Boyacá, tal como se muestra en el Tabla I.

Tabla I. Temperatura de la mezcla $-\mathrm{Tmix}{ }^{\circ} \mathrm{C}$

\begin{tabular}{|c|c|}
\hline $\begin{array}{c}\text { Temperatura media anual } \\
\text { ponderada del aire }-\mathbf{T M A P},{ }^{\circ} \mathbf{C}\end{array}$ & $\begin{array}{c}\text { Temperatura de la } \\
\text { mezcla }- \text { Tmix }{ }^{\circ} \mathbf{C}\end{array}$ \\
\hline 13 & 19.6 \\
\hline 14 & 21.0 \\
\hline 15 & 22.5 \\
\hline 16 & 24.0 \\
\hline 17 & 25.4 \\
\hline 18 & 26.9 \\
\hline 19 & 28.3 \\
\hline 20 & 29.7 \\
\hline
\end{tabular}

Fuente: Elaboración propia.

\subsection{Tiempo de aplicación de la carga - t. El} tiempo de aplicación de la carga del tránsito representa el efecto dinámico de operación de una estructura de un pavimento y se determina mediante (7), la cual es función del espesor probable de la capa asfáltica y de la velocidad de operación [1].

$t=10^{(0.005 \text { hasf }-0.2-0.94 \log (\text { Vop }))}$

Donde:

$t \quad=$ Tiempo de aplicación de la carga, $s$

hasf $=$ Espesor probable de la capa asfáltica, cm (se consideró para la investigación de $10 \mathrm{~cm}$ )

Vop $=$ Velocidad de operación, Km/hora
2.5 Frecuencia de aplicación de la carga - F. La frecuencia de aplicación de la carga define el efecto dinámico de la carga a la estructura del pavimento y está asociado al tiempo de aplicación de la carga como se muestra en (8). [3]

$F=\frac{1}{2 \pi t}$

Donde:

$F=$ Frecuencia de aplicación de la carga, $\mathrm{Hz}$

$t$ = Tiempo de aplicación de la carga, $s$

En el Tabla II se muestra los valores de las variables velocidad de operación, tiempo y frecuencia de aplicación de la carga, utilizados en este trabajo de investigación.

Tabla II. Variables dinámicas de aplicación de la carga

\begin{tabular}{|c|c|c|}
\hline $\begin{array}{c}\text { Velocidad de operación - } \\
\text { Vop, Km/hora }\end{array}$ & $\begin{array}{c}\text { Tiempo de aplicación de } \\
\text { la carga } \mathbf{- t ,} \mathbf{s}\end{array}$ & $\begin{array}{c}\text { Frecuencia de aplicación } \\
\text { de la carga } \mathbf{- ~ F , ~ H z}\end{array}$ \\
\hline 30 & 0.029 & 5.5 \\
\hline 50 & 0.018 & 8.8 \\
\hline 70 & 0.013 & 12.2 \\
\hline 100 & 0.009 & 17.7 \\
\hline
\end{tabular}

Fuente: Elaboración propia. 
2.6 Caracterización de los cementos asfálticos. Se analizaron en laboratorio dos tipos de asfaltos de uso frecuente en la construcción de carreteras del departamento de Boyacá, el asfalto AC 60 - 70 y el asfalto
AC $80-100$. Las propiedades del asfalto se presentan en el Tabla III. El índice de penetración se determinó utilizando el programa BAND 2.0 de la Shell [4].
Enero - Junio 2016 ISSN 0122-820X E-ISSN 2422-5053 PP: 108-119

Tabla III. Propiedades del asfalto

\begin{tabular}{|c|c|c|c|}
\hline NORMA & ENSAYO & AC 80 - 100 & AC 60 - 70 \\
\hline INV E - 706 - 13 & Penetración $\left(25^{\circ} \mathrm{C} .100 \mathrm{~g} .5 \mathrm{~s}\right)$ & 83 & 65 \\
\hline INV E - 723 - 13 & Punto de inflamación, ${ }^{\circ} \mathrm{C}$ & 241 & 245 \\
\hline INV E - 707 - 013 & Gravedad Especifica & 1.069 & 1.012 \\
\hline INV E - 712 - 13 & Punto de ablandamiento-anillo y bola, ${ }^{\circ} \mathrm{C}$ & 48 & 53 \\
\hline INV E - 714 - 13 & Viscosidad Saybolt Furol (P) & 1050 & 1650 \\
\hline INV E - 724 - 13 & Índice de Penetración & -0.40 & +0.20 \\
\hline
\end{tabular}

Fuente: Elaboración propia.

2.7 Módulo dinámico del asfalto - Sasf. El módulo dinámico del asfalto se determinó teniendo en cuenta la temperatura media anual ponderada del aire - TMAP, la temperatura de la mezcla-Tmix, el tiempo de aplicación de la carga - $t$, la frecuencia de aplicación de la carga - F, la diferencia de temperatura - DT y el índice de penetración - IP del asfalto. Para la presente investigación el módulo dinámico del asfalto se calculó con el programa BAND 2.0 de la Shell.

\subsection{Módulo dinámico de la mezcla asfáltica}

- Smix. El módulo dinámico de la mezcla asfáltica se determinó teniendo en cuenta los parámetros volumétricos de la mezcla asfáltica densa en caliente tipo MDC-19 para rodadura, Artículo 450-13 del INVIAS [5], la cual es una mezcla típica que se fabrica en el departamento de Boyacá.

Los parámetros volumétricos promedios de la mezcla asfáltica densa en caliente tipo MDC19 para rodadura (según la metodología de diseño de mezclas asfálticas Marshall Modificado), diseñada con agregados típicos de la región de Tunja son las siguientes:

- Porcentaje del volumen de vacíos, Vv $=4.0 \%$

- Porcentaje del volumen de asfalto de la mezcla, $\mathrm{Vb}=11 \%$

- Porcentaje del volumen de agregados de la mezcla, Vag $=85 \%$
Para la presente investigación el módulo dinámico de la mezcla asfáltica se calculó con el programa BAND 2.0 de la Shell.

Es importante destacar que la simulación o estimación del módulo dinámico se realizó con el objeto de analizar su comportamiento dinámico ante los cambios de las condiciones de trabajo y no se realizaron ensayos de laboratorio porque el número de ensayos es muy grande.

\section{Resultados y análisis}

3.1 Influencia de la temperatura media anual ponderada del aire - TMAP y la velocidad de operación en el módulo dinámico del asfalto. La temperatura tiene influencia directa en la viscosidad del asfalto y por tanto en su módulo dinámico, tal como se muestra en las Figuras 2 y 3 , siendo su tendencia inversamente proporcional, a mayor temperatura menor módulo dinámico del asfalto, es decir que el asfalto es más blando o menos viscoso. [6]. 
ISSN $0122-820 \mathrm{X}$

E-ISSN 2422-5053 PP: 108-119
Efecto de la temperatura en el módulo dinámico del cemento asfáltico y la mezcla

asfáltica - caso de Boyacá

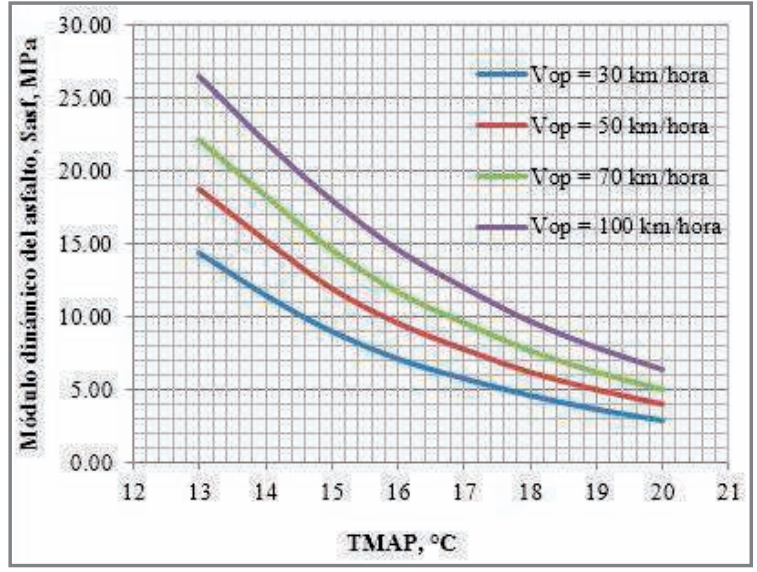

Asfalto AC 60 - 70

Figura 2 y 3. Comportamiento del módulo dinámico del asfalto en función de la temperatura y la velocidad de operación. Asfalto AC 60 - 70.
La Figura 2 muestra el comportamiento del módulo dinámico del asfalto $\mathrm{AC} 60$ - 70 en función de la temperatura media anual ponderada del aire - TMAP para las velocidades de operación de 30,50, 70 y $100 \mathrm{Km} /$ hora y la Figura 3 muestra el comportamiento del asfalto AC $80-100$. Del análisis de estas figuras se deduce lo siguiente:

- La tendencia del módulo dinámico del asfalto en función de la temperatura es decreciente e inversamente proporcional.

- Para velocidades de operación bajas el módulo dinámico del asfalto es bajo y a medida que se incrementa la velocidad de operación el módulo dinámico del asfalto aumenta.

- Al comparar el comportamiento del módulo dinámico del asfalto en función de la temperatura media anual ponderada del aire, para los asfaltos AC $60-70$ y AC $80-100$, se tiene: Para asfaltos de mayor consistencia (AC 60 - 70) el módulo dinámico del asfalto es mayor que el obtenido para la misma temperatura con un asfalto de menor consistencia (AC 80 - 100), tal como se aprecia en los Tablas IV, V y en las Figuras 2 y 3.
- Para temperaturas bajas, el módulo dinámico del asfalto es más sensible que para temperaturas altas, tal como se muestra en las Figuras 2 y 3.

- Al comparar el módulo dinámico del asfalto en función de la velocidad de operación, (ver Figuras 2 y 3), se tiene que a medida que aumenta la velocidad de operación la variación del módulo dinámico del asfalto por grado de temperatura $\mathrm{MPa} /{ }^{\circ} \mathrm{C}$ es mayor, tal como se muestra en el Tabla VI; lo anterior, permite analizar la sensibilidad de una curva de módulo dinámico del asfalto en función de la temperatura para una velocidad de operación considerada. El análisis se realizó para los asfaltos AC 60-70 y AC 80-100.

En los Tablas IV y V se presenta el efecto de la temperatura en el módulo dinámico del cemento asfáltico tipo AC $60-70$ y AC $80-$ $100 \mathrm{y}$ se destaca las siguientes tendencias 
Tabla IV. Efecto de la temperatura en el módulo dinámico del asfalto y de la mezcla asfáltica, AC 60 - 70

\begin{tabular}{|l|c|c|c|c|c|c|}
\hline $\begin{array}{c}\text { Temperatura } \\
\text { media anual } \\
\text { ponderada - }\end{array}$ & $\begin{array}{c}\text { Temperatura } \\
\text { de la mezcla }- \\
\text { Tmix, }{ }^{\circ} \mathrm{C}\end{array}$ & $\begin{array}{c}\text { Velocidad de } \\
\text { operación, } \\
\mathrm{Km} / \mathrm{hora}\end{array}$ & $\begin{array}{c}\text { Tiempo de } \\
\text { aplicación de } \\
\text { la carga }-\mathrm{t}, \mathrm{s}\end{array}$ & $\begin{array}{c}\text { Frecuencia de } \\
\text { aplicación de } \\
\text { la carga }-\mathrm{F}, \mathrm{Hz}\end{array}$ & $\begin{array}{c}\text { Temperatura de } \\
\text { penetración del } \\
\text { asfalto, }{ }^{\circ} \mathrm{C}\end{array}$ & $\begin{array}{c}\text { Penetración } \\
\text { del asfalto a } \\
25^{\circ} \mathrm{C}, 1 / 10\end{array}$ \\
\hline
\end{tabular}

Temperatura Indice de

Ablandamiento Penetración $\mathrm{mm}$

\begin{tabular}{|c|c|} 
TMAP, ${ }^{\circ} \mathrm{C}$ & Tmix $^{\circ} \mathrm{C}$ \\
\hline 13 & 19.6 \\
\hline 14 & 21.0 \\
\hline
\end{tabular}

\begin{tabular}{|l|l|}
\hline 13 & 19.6 \\
\hline 15 & 21.0 \\
\hline 16 & 22.5 \\
\hline
\end{tabular}

\begin{tabular}{|l|l|}
\hline 17 & 24.0 \\
\hline 18 & 25.4 \\
\hline 19 & 26.9 \\
\hline
\end{tabular}

carga $-t, s$ la carga $-F$,

asfalto, ${ }^{\circ} \mathrm{C}$

(n)

30

\begin{tabular}{l|l} 
& \\
0.029 & 5.5 \\
& \\
&
\end{tabular}

del asfalto, ${ }^{\circ} \mathrm{C}$

\begin{tabular}{l|l} 
& \\
\hline
\end{tabular}

IP

Módulo del Módulo de la asfalto - Sasf, mezcla asfáltica -

(2)

\begin{tabular}{|l|r|}
\hline 20 & 29.7 \\
\hline 13 & 19.6 \\
\hline
\end{tabular}

\begin{tabular}{|l|l|}
\hline 13 & 19.6 \\
\hline 14 & 21.0 \\
\hline 15 & 22.5 \\
\hline
\end{tabular}

\begin{tabular}{|l}
15 \\
\hline 16 \\
\hline 17
\end{tabular}

18

18

\begin{tabular}{|l|l|}
\hline & 28.3 \\
\hline 20 & 29.7 \\
\hline
\end{tabular}

\begin{tabular}{|l|r|}
\hline 13 & 19.6 \\
\hline 14 & 21.0 \\
\hline
\end{tabular}

\begin{tabular}{|l|l|}
\hline 14 & 21.0 \\
\hline 15 & 22.5 \\
\hline 16 & 24.0 \\
\hline
\end{tabular}

\begin{tabular}{|l|l|}
\hline 16 & 24.0 \\
\hline 17 & 25.4 \\
\hline
\end{tabular}

\begin{tabular}{|l|l|}
\hline 18 & 25.4 \\
\hline 19 & 26.9 \\
\hline 20 & 28.3 \\
\hline
\end{tabular}

\begin{tabular}{l|l|l}
20 & 29.7 & \\
\hline
\end{tabular}

\begin{tabular}{|l|l|}
\hline 13 & 19.6 \\
\hline
\end{tabular}

\begin{tabular}{|l|l|}
\hline 13 & 19.6 \\
\hline 15 & 21.0 \\
\hline 15 & 22.5 \\
\hline 16 & 24.0 \\
\hline 17 & 25.4 \\
\hline 18 & 26.9 \\
\hline 19 & 28.3 \\
\hline 20 & 29.7 \\
\hline
\end{tabular}

100

0.009

\section{\begin{tabular}{|l|l|l|l}
\hline & &
\end{tabular}

\begin{tabular}{|l|l|l|}
\hline & & \\
0.018 & 8.8 & 25 \\
& & \\
\hline
\end{tabular}

(1)

0.2

$\mathrm{MPa}$

Smix, $\mathrm{MPa}$

\begin{tabular}{|c|c|}
\hline 14.40 & 3010 \\
\hline 11.50 & 2600 \\
\hline 9.01 & 2220 \\
\hline 7.13 & 1910 \\
\hline 5.77 & 1660 \\
\hline 4.61 & 1350 \\
\hline 3.66 & 1100 \\
\hline 2.89 & 850 \\
\hline
\end{tabular}

\begin{tabular}{|c|c|}
\hline 18.80 & 3580 \\
\hline 15.30 & 3130 \\
\hline 12.00 & 2680 \\
\hline 9.62 & 2320 \\
\hline 7.83 & 2030 \\
\hline 6.25 & 1750 \\
\hline 5.06 & 1530 \\
\hline 4.07 & 1250 \\
\hline
\end{tabular}

70
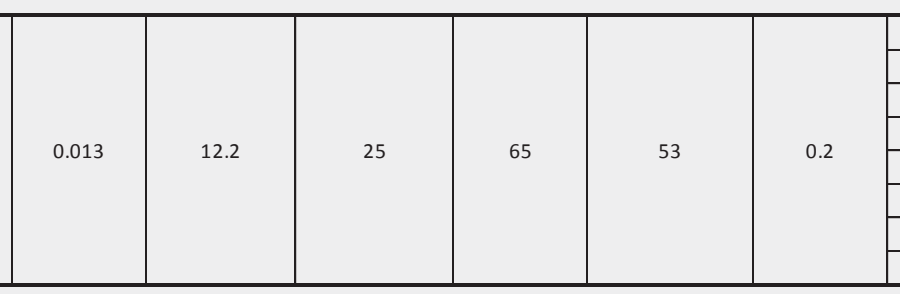

\begin{tabular}{|c|c|}
\hline 22.10 & 3980 \\
\hline 18.30 & 3520 \\
\hline 14.60 & 3040 \\
\hline 11.70 & 2630 \\
\hline 9.60 & 2310 \\
\hline 7.69 & 2000 \\
\hline 6.23 & 1750 \\
\hline 5.04 & 1520 \\
\hline
\end{tabular}

0.009

17.7

Fuente: Elaboración propia.

3.2 Influencia de la temperatura media anual ponderada - TMAP y la velocidad de operación en el módulo dinámico de la mezcla asfáltica. El módulo dinámico de las mezclas asfálticas depende de la temperatura y de las condiciones dinámicas de la mezcla asfáltica, tal como se muestra en los Tablas V, VI y en las Figuras 4 y 5.

Enero - Junio 2016 
Efecto de la temperatura en el módulo dinámico del cemento asfáltico y la mezcla asfáltica - caso de Boyacá

Tabla V. Efecto de la temperatura en el módulo dinámico del asfalto y de la mezcla asfáltica, AC 80 - 100

\begin{tabular}{|c|c|c|c|c|c|c|c|c|c|c|}
\hline $\begin{array}{c}\text { Temperatura } \\
\text { media anual } \\
\text { ponderada - } \\
T M A P,{ }^{\circ} \mathrm{C}\end{array}$ & $\begin{array}{c}\text { Temperatura } \\
\text { de la mezcla - } \\
\text { Tmix, }{ }^{\circ} \mathrm{C}\end{array}$ & $\begin{array}{c}\text { Velocidad de } \\
\text { operación, } \\
\text { Km/hora }\end{array}$ & $\begin{array}{c}\text { Tiempo de } \\
\text { aplicación de } \\
\text { la carga - } t, s\end{array}$ & $\begin{array}{c}\text { Frecuencia de } \\
\text { aplicación de } \\
\text { la carga }-\mathrm{F}, \mathrm{Hz}\end{array}$ & $\begin{array}{c}\text { Temperatura de } \\
\text { penetración del } \\
\text { asfalto, }{ }^{\circ} \mathrm{C}\end{array}$ & $\begin{array}{c}\text { Penetración } \\
\text { del asfalto a } \\
25^{\circ} \mathrm{C}, 1 / 10 \\
\mathrm{~mm}\end{array}$ & $\begin{array}{c}\text { Temperatura } \\
\text { Ablandamiento } \\
\text { del asfalto, }{ }^{\circ} \mathrm{C}\end{array}$ & $\begin{array}{c}\text { Indice de } \\
\text { Penetración, } \\
\text { IP }\end{array}$ & $\begin{array}{c}\text { Módulo del } \\
\text { asfalto - Sasf, } \\
\text { MPa }\end{array}$ & $\begin{array}{c}\text { Módulo de la } \\
\text { mezcla asfáltica } \\
\text { Smix, MPa }\end{array}$ \\
\hline 13 & 19.6 & \multirow{8}{*}{30} & \multirow{8}{*}{0.029} & \multirow{8}{*}{5.5} & \multirow{8}{*}{25} & \multirow{8}{*}{83} & \multirow{8}{*}{48} & \multirow{8}{*}{-0.4} & 11.60 & 2620 \\
\hline 14 & 21.0 & & & & & & & & 9.09 & 2230 \\
\hline 15 & 22.5 & & & & & & & & 7.01 & 1890 \\
\hline 16 & 24.0 & & & & & & & & 5.30 & 1570 \\
\hline 17 & 25.4 & & & & & & & & 4.08 & 1250 \\
\hline 18 & 26.9 & & & & & & & & 3.07 & 1000 \\
\hline 19 & 28.3 & & & & & & & & 2.36 & 750 \\
\hline 20 & 29.7 & & & & & & & & 1.85 & 500 \\
\hline 13 & 19.6 & \multirow{8}{*}{50} & \multirow{8}{*}{0.018} & \multirow{8}{*}{8.8} & \multirow{8}{*}{25} & \multirow{8}{*}{83} & \multirow{8}{*}{48} & \multirow{8}{*}{-0.4} & 15.60 & 3170 \\
\hline 14 & 21.0 & & & & & & & & 12.60 & 2760 \\
\hline 15 & 22.5 & & & & & & & & 9.80 & 2350 \\
\hline 16 & 24.0 & & & & & & & & 7.57 & 1980 \\
\hline 17 & 25.4 & & & & & & & & 5.86 & 1680 \\
\hline 18 & 26.9 & & & & & & & & 4.43 & 1350 \\
\hline 19 & 28.3 & & & & & & & & 3.42 & 1050 \\
\hline 20 & 29.7 & & & & & & & & 2.67 & 750 \\
\hline 13 & 19.6 & \multirow{8}{*}{70} & \multirow{8}{*}{0.013} & \multirow{8}{*}{12.2} & \multirow{8}{*}{25} & \multirow{8}{*}{83} & \multirow{8}{*}{48} & \multirow{8}{*}{-0.4} & 18.90 & 3590 \\
\hline 14 & 21.0 & & & & & & & & 15.40 & 3150 \\
\hline 15 & 22.5 & & & & & & & & 12.30 & 2720 \\
\hline 16 & 24.0 & & & & & & & & 9.50 & 2300 \\
\hline 17 & 25.4 & & & & & & & & 7.47 & 1970 \\
\hline 18 & 26.9 & & & & & & & & 5.67 & 1640 \\
\hline 19 & 28.3 & & & & & & & & 4.39 & 1250 \\
\hline 20 & 29.7 & & & & & & & & 3.45 & 950 \\
\hline 13 & 19.6 & \multirow{8}{*}{100} & \multirow{8}{*}{0.009} & \multirow{8}{*}{17.7} & \multirow{8}{*}{25} & \multirow{8}{*}{83} & \multirow{8}{*}{48} & & 23.70 & 4160 \\
\hline 15 & 21.0 & & & & & & & & 19.20 & 3630 \\
\hline 15 & 22.5 & & & & & & & & 15.40 & 3150 \\
\hline 16 & 24.0 & & & & & & & 0 & 12.20 & 2700 \\
\hline 17 & 25.4 & & & & & & & -0.4 & 9.65 & 2320 \\
\hline 18 & 26.9 & & & & & & & & 7.46 & 1960 \\
\hline 19 & 28.3 & & & & & & & & 5.80 & 1670 \\
\hline 20 & 29.7 & & & & & & & & 4.57 & 1350 \\
\hline
\end{tabular}

Fuente: Elaboración propia.

TABLA VI. Análisis de sensibilidad de las curvas del módulo dinámico del asfalto en función de la velocidad de operación

\begin{tabular}{|c|c|c|c|c|c|c|}
\hline \multirow[b]{2}{*}{$\begin{array}{c}\text { Velocidad de } \\
\text { operación } \\
\text { Vop, Km/hora }\end{array}$} & \multicolumn{3}{|c|}{ Asfalto AC 60 - 70} & \multicolumn{3}{|c|}{ Asfalto AC 80-100 } \\
\hline & $\begin{array}{c}\text { Diferencia de } \\
\text { temperatura } \\
\Delta^{\circ} \mathrm{C}\end{array}$ & $\begin{array}{c}\text { Diferencia del } \\
\text { módulos del } \\
\text { asfalto- } \Delta \text { Sasf, } \\
\text { MPa }\end{array}$ & $\begin{array}{c}\text { Variación - } \\
\Delta \mathrm{Sasf} / \Delta^{\circ} \mathrm{C}, \\
\mathrm{MPa} /{ }^{\circ} \mathrm{C}\end{array}$ & $\begin{array}{c}\text { Diferencia de } \\
\text { temperatura } \Delta \\
{ }^{\circ} \mathrm{C}\end{array}$ & $\begin{array}{c}\text { Diferencia del } \\
\text { módulos del } \\
\text { asfalto- } \Delta \text { Sasf, } \\
\mathrm{MPa}\end{array}$ & $\begin{array}{c}\text { Variación - } \\
\Delta \mathrm{Sasf} / \Delta^{\circ} \mathrm{C}, \\
\mathrm{MPa} /{ }^{\circ} \mathrm{C}\end{array}$ \\
\hline 30 & \multirow{4}{*}{7} & 11.51 & 1.64 & \multirow{4}{*}{7} & 9.75 & 1.39 \\
\hline 50 & & 14.73 & 2.10 & & 12.93 & 1.85 \\
\hline 70 & & 17.06 & 2.43 & & 15.45 & 2.21 \\
\hline 100 & & 20.12 & 2.87 & & 19.13 & 2.73 \\
\hline
\end{tabular}

Fuente: Elaboración propia.

- Del análisis de la información contenida en los Tablas IV, V y las Figuras 4 y 5 se deduce lo siguiente:

- La tendencia del módulo dinámico de la mezcla asfáltica en función de la temperatura es decreciente e inversamente proporcional.

- Para velocidades de operación bajas el módulo dinámico de la mezcla asfáltica es bajo 
y a medida que se incrementa la velocidad de operación el módulo dinámico de la mezcla aumenta significativamente.

- Al comparar el comportamiento del módulo dinámico de la mezcla asfáltica en función de la temperatura para los asfaltos AC $60-70$ y AC 80 - 100, se tiene: para asfaltos de mayor consistencia (AC 60 - 70) el módulo dinámico de la mezcla asfáltica es mayor (Ver Figura 4) que el obtenido para la misma temperatura con un asfalto de menor consistencia (AC 80 -100), tal como se aprecia en la Figura 5. [7], [8].

Para temperaturas bajas, el módulo dinámico de la mezcla asfáltica es más sensible que para temperaturas altas. (Ver Figuras 4 y 5).

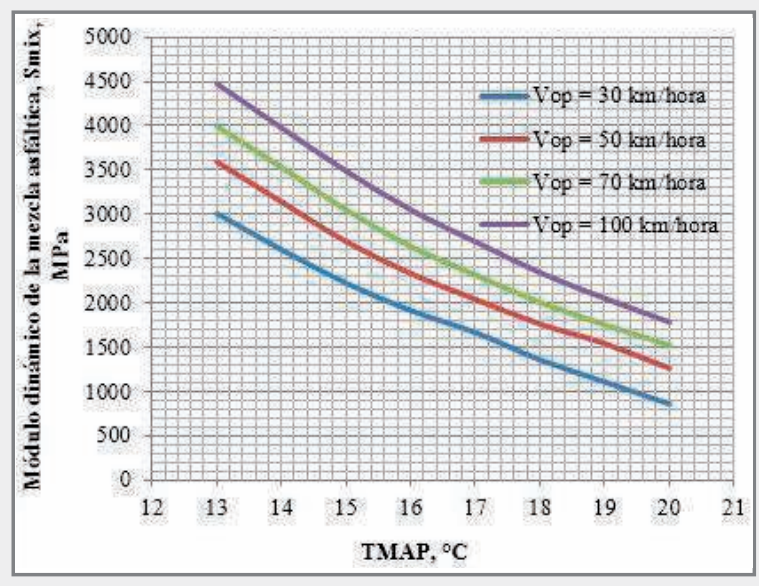

AC $60-70$

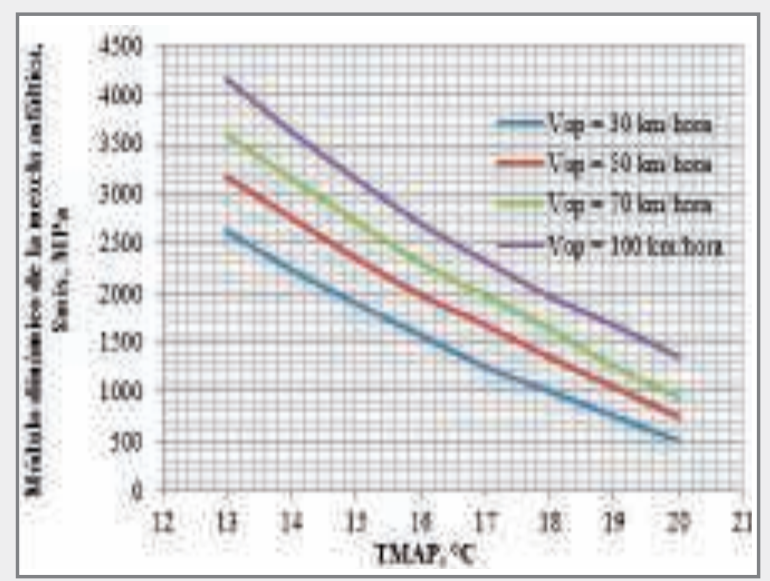

AC $80-100$

Figura 4 y 5. Comportamiento del módulo dinámico de la mezcla asfáltica en función de la temperatura y la velocidad de operación. Asfalto AC $60-70$, AC $80-100$

Fuente: Elaboración propia.

- Al comparar el módulo dinámico de la mezcla asfáltica en función de la velocidad de operación, (Ver Figuras 4 y 5), se tiene que a medida que aumenta la velocidad de operación la variación del módulo dinámico de la mezcla asfáltica por grado de temperatura $\mathrm{MPa} /{ }^{\circ} \mathrm{C}$ es mayor, tal como se muestra en el Tabla VII; lo anterior, permite analizar la sensibilidad de una curva de módulo dinámico de la mezcla asfáltica en función de la temperatura para una velocidad de operación considerada. El análisis se realizó para los asfaltos AC 60-70 y AC 80-100.

Tabla VII. Análisis de sensibilidad de las curvas del módulo dinámico de la mezcla asfáltica en función de la velocidad de operación

\begin{tabular}{|c|c|c|c|c|c|c|}
\hline $\begin{array}{c}\text { Velocidad de } \\
\text { operación } \\
\text { Vop, Km/hora }\end{array}$ & $\begin{array}{c}\text { Diferencia de } \\
\text { temperatura } \\
\Delta^{\circ} \mathrm{C}\end{array}$ & $\begin{array}{c}\text { Diferencia del } \\
\text { módulo de la } \\
\text { mezcla asfáltica- } \\
\Delta \text { Smix, MPa }\end{array}$ & $\begin{array}{c}\text { Variación - } \\
\Delta \mathrm{Smix} / \Delta^{\circ} \mathrm{C}, \\
\mathrm{MPa} /{ }^{\circ} \mathrm{C}\end{array}$ & $\begin{array}{c}\text { Diferencia de } \\
\text { temperatura } \\
\Delta^{\circ} \mathrm{C}\end{array}$ & $\begin{array}{l}\text { Diferencia del } \\
\text { módulo de la mezcla } \\
\text { asfáltica- } \Delta \text { Smix, } \\
\text { MPa }\end{array}$ & $\begin{array}{c}\text { Variación - } \\
\Delta \mathrm{Smix} / \Delta^{\circ} \mathrm{C}, \\
\mathrm{MPa} /{ }^{\circ} \mathrm{C}\end{array}$ \\
\hline 50 & \multirow{3}{*}{7} & 2330 & 332.86 & \multirow{3}{*}{7} & 2420 & 345.71 \\
\hline 70 & & 2460 & 351.43 & & 2640 & 377.14 \\
\hline 100 & & 2700 & 385.71 & & 2810 & 401.43 \\
\hline
\end{tabular}


No. 1

Enero - Junio 2016 ISSN 0122-820X E-ISSN 2422-5053 PP: 108-119

\section{Conclusiones}

\subsection{Efecto de la temperatura media anual} ponderada del aire en el módulo dinámico del asfalto

- La tendencia del módulo dinámico del asfalto en función de la temperatura es decreciente e inversamente proporcional.

- Para velocidades de operación bajas el módulo dinámico del asfalto es bajo y a medida que se incrementa la velocidad de operación el módulo dinámico del asfalto aumenta.

- Al comparar el comportamiento del módulo dinámico del asfalto en función de la temperatura media anual ponderada del aire, para los asfaltos AC $60-70$ y AC $80-100$, se tiene: Para asfaltos de mayor consistencia (AC 60 - 70) el módulo dinámico del asfalto es mayor que el obtenido para la misma temperatura con un asfalto de menor consistencia (AC $80-100)$.

- Para temperaturas bajas, el módulo dinámico del asfalto es más sensible que para temperaturas altas.

- Al comparar el módulo dinámico del asfalto en función de la velocidad de operación, se tiene que a medida que aumenta la velocidad de operación la variación del módulo dinámico del asfalto por grado de temperatura $\mathrm{MPa} /{ }^{\circ} \mathrm{C}$ es mayor.

\subsection{Efecto de la temperatura media anual ponderada del aire en el módulo dinámico de la mezcla asfáltica}

- La tendencia del módulo dinámico de la mezcla asfáltica en función de la temperatura es decreciente e inversamente proporcional.

- Para velocidades de operación bajas el módulo dinámico de la mezcla asfáltica es bajo y a medida que se incrementa la velocidad de operación el módulo dinámico de la mezcla aumenta significativamente.
- Al comparar el comportamiento del módulo dinámico de la mezcla asfáltica en función de la temperatura para los asfaltos AC $60-$ 70 y AC $80-100$, se tiene: Para asfaltos de mayor consistencia (AC 60 - 70) el módulo dinámico de la mezcla asfáltica es mayor que el obtenido para la misma temperatura con un asfalto de menor consistencia (AC 80 - 100).

- Para temperaturas bajas, el módulo dinámico de la mezcla asfáltica es más sensible que para temperaturas altas

- Al comparar el módulo dinámico de la mezcla asfáltica en función de la velocidad de operación, se tiene que a medida que aumenta la velocidad de operación la variación del módulo dinámico de la mezcla asfáltica por grado de temperatura $\mathrm{MPa} /{ }^{\circ} \mathrm{C}$ es mayor.

\section{Agradecimientos}

El autor expresa sus agradecimientos a la Escuela de Transporte y Vías de la Facultad de Ingeniería de la Universidad Pedagógica y Tecnológica de Colombia por el apoyo brindado al desarrollo de este proyecto de investigación.

\section{Referencias}

[1] SHELL. Manual del usuario del programa SHELL, módulo BISAR 3.0.

[2] C. H, Higuera Sandoval. Nociones sobre métodos de diseño de estructuras de pavimentos para carreteras. Volumen I y II. Universidad Pedagógica y Tecnológica de Colombia. ISBN 978-958-660-149-8 y ISBN 978-958-660-152-8, Tunja. 2011.

[3] Y. H, Huang. Pavement analysis and design. Prentice - Hall. Inc. United State of America. 2004.

[4] Shell, Guía de diseño de pavimentos flexibles por el método SHELL. Programa de cálculo - SPDM-PC. 2000.

[5] Instituto Nacional de Vías - INVIAS. Especificaciones generales de 
construcción de carreteras. Bogotá. 2013.

[6] F. J, Lilli. Curso sobre diseño racional de pavimentos. Instituto de Postgrado en Vías e Ingeniería Civil. Universidad del Cauca. Popayán. 1987.

[7] F. A, Reyes Lizcano. Diseño racional de pavimentos. Bogotá. Editorial Ceja y la Escuela Colombiana de Ingeniería. 2004.

[8] American Association Of State Highway Transportation Official. Guide for Design of Pavement Structures 1993. 